\title{
Experimental investigation on air entrainment below impinging jets by means of video observations and image processing
}

\author{
D. V. Danciu, M. J. da Silva, M. Schmidtke, D. Lucas \\ \& U. Hampel \\ Institute of Safety Research, \\ Forschungszentrum Dresden-Rossendorf e.V., Germany
}

\begin{abstract}
Air entrainment as a result of a water jet plunging into a pool of water was studied by means of visualization techniques. Image processing algorithms were used to analyze the recorded sequences of the phenomenon. Data regarding the penetration depth and bubble size distribution was gathered for different jet impact velocities and jet lengths. The accumulated results are further used to validate the models implemented in computational fluid dynamics (CFD) codes.

Keywords: air entrainment, plunging jet, penetration depth, image processing, bubbles size distribution.
\end{abstract}

\section{Introduction}

The phenomenon of air entrainment as a result of an impinging jet plunging into a pool of water occurs in many industrial processes (steel industry, wastewater treatment, food industry), as well as in nature (cascades, breaking waves).

A water jet impinging on a free surface of a pool causes air entrainment as soon as the jet velocity is high enough (inception velocity). A swarm of bubbles appears as result of the impingement. Impinging jets are also important in different scenarios considered in nuclear reactor safety analyses. One example is the emergency core cooling (ECC) injection into a cold leg partially filled with steam and water. Such a situation is accounted in some scenarios of loss of coolant accidents. In this case, the injected cold water impinges as a jet on the surface of the hot water. Depending on the velocity of the jet, steam bubbles may 
be entrained below the surface by the impinging jet. These bubbles contribute to heat exchange and mixing of the fluids. Heat transfer between cold and hot water and mixing in the cold leg play an important role since the mixed water enters the reactor pressure vessel and may cause high temperature gradients at the wall of the vessel (pressurized thermal shock).

The mechanism of air entrainment has been studied over the past years by different researchers. Recent works in this area are those of Chirichella et al. [5] and Cummings and Chanson [7]. The experiments described in [5] studied air entrainment produced by a translating axisymmetric laminar water jet and distinguished between four entrainment regimes. In [6] air entrainment was studied by means of visualization experiments. The inception conditions of airwater flows were found to depend critically upon the jet turbulence. Air entrainment was also observed to be caused by the presence of foam bubbles at the jet-plunge pool intersection. In the experimental study described in [1], the entrainment regimes are studied and a proper nozzle configuration is being searched for, in order to minimize the turbulence effects of the jet.

Oguz [11] experimentally and theoretically studied the role of the surface disturbances in the entrainment of bubbles. He showed that a key aspect of the process is the jet roughness. The results gathered from the study show the importance of the jet surface properties, in contrast to previous investigations. The average roughness of the jet was discovered to be controlling the air entrainment rate and the average wavelength of the disturbances to be correlated to the bubble size distribution.

In a theoretical and experimental study on air entrainment [11] is showed that an undisturbed jet does not entrap air even at relatively high Reynolds numbers.

Some studies have been made regarding air entrainment caused by means of impinging jets. We found though there is a certain lack of systematic data in the literature with regard to the penetration depth of the bubbles and the gas void fraction.

We developed our experimental setup to study the air entrainment phenomenon under different velocity and jet length conditions. Main goal is to gain experimental data to validate the models implemented in the computational fluid dynamics (CFD) codes.

\section{Experimental setup}

Figure 1 shows a schematic of the experimental setup. The experiments were carried out in a $0.3 \mathrm{~m} \times 0.3 \mathrm{~m} \times 0.5 \mathrm{~m}$ water tank constructed with transparent acrylic walls for visualization purposes. The water level in the tank was kept constant at $0.28 \mathrm{~m}$ throughout the experiments. Water was pumped out of the tank and re-injected through a smooth $6 \mathrm{~mm}$ diameter, $50 \mathrm{~mm}$ length steel pipe used as nozzle to produce a vertical falling round jet. A rotameter was used for the measurement of the flow rate. Images of the impact between the jet and the water pool were captured by a high-speed camera (DRS Technologies). For each experimental condition, a sequence of images of the region below the surface was taken. The camera was operated with a frame rate of 200 frames per second. 
Backlighting with high luminosity LED panels was used during the experiments in order to have a proper exposure at the required filming rate.

Experiments were realized for different nozzle heights $\left(\mathrm{L}_{j}\right.$ in Fig. 1) and volumetric flow rates.

The experiments consisted in a vertical liquid jet of different lengths and velocities impinging on the calm surface of the water pool at an angle of $90^{\circ}$. The velocity of the jet ranged between $0.9 \mathrm{~m} / \mathrm{s}$ and $2.5 \mathrm{~m} / \mathrm{s}$ at the nozzle exit and the jet length was varied between $0.01 \mathrm{~m}$ and $0.2 \mathrm{~m}$. The test matrix is shown in Table 1.

The velocity of the jet at the plunge point is calculated as:

$$
v_{j}=\sqrt{v_{0}^{2}+2 g L_{j}}
$$

where $\mathrm{v}_{0}$ is the velocity of the jet at the nozzle exit, $L_{\mathrm{j}}$ is the jet length and $g$ the earth acceleration. In (1) free falling is assumed for the water after leaving the nozzle.

\section{Preliminary observations}

In some cases it is difficult to determine the conditions for the inception of air entrainment. Air entrainment can also occur for a very short period of time immediately after the impact, regardless of the nozzle height and the velocity of the jet.

Similar to [5], where it was stated that the incipient air entrainment takes place with the appearance of at least one bubble, in the absence of other bubbles in the water pool, which lasts longer than $2 \mathrm{~s}$ beneath the surface of the pool, we also observed three different regimes during our experiments.

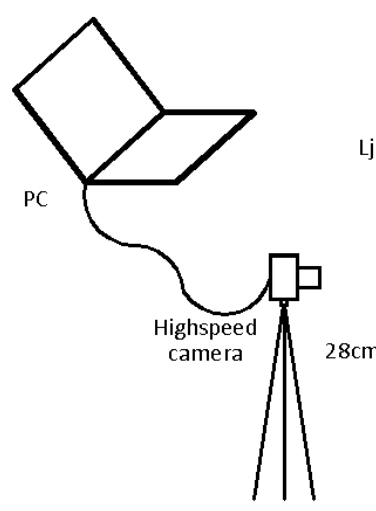

Figure 1:

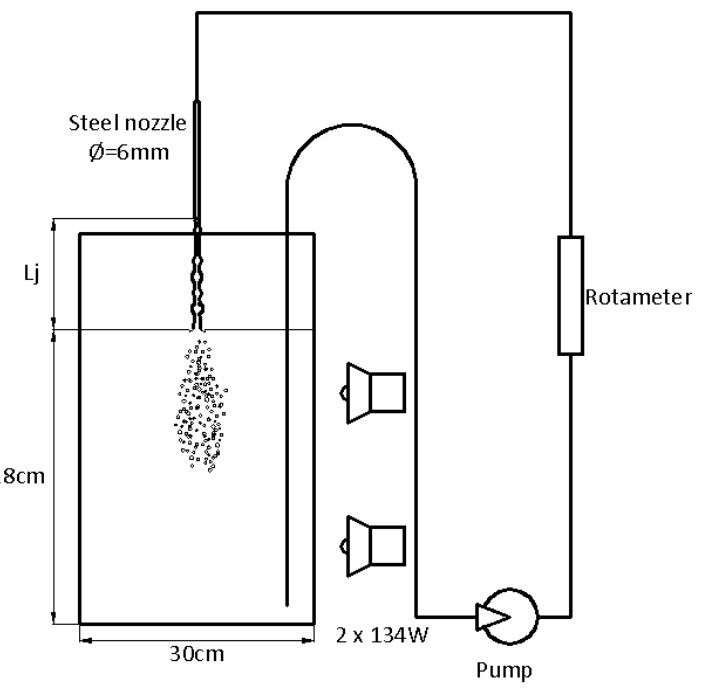


Table 1: $\quad$ Test matrix.

\begin{tabular}{|c|c|c|c|c|c|c|c|c|c|c|}
\hline \multicolumn{2}{|c|}{$\mathrm{L}_{\mathrm{j}}[\mathrm{m}]$} & $\begin{array}{c}0.01 \\
0 \\
\end{array}$ & $\begin{array}{c}0.02 \\
5\end{array}$ & $\begin{array}{c}0.05 \\
0 \\
\end{array}$ & $\begin{array}{c}0.07 \\
5\end{array}$ & $\begin{array}{c}0.10 \\
0\end{array}$ & $\begin{array}{c}0.12 \\
5\end{array}$ & $\begin{array}{c}0.15 \\
0\end{array}$ & $\begin{array}{c}0.17 \\
5\end{array}$ & $\begin{array}{c}0.20 \\
0\end{array}$ \\
\hline $\begin{array}{c}\mathrm{v}_{0} \\
{[\mathrm{~m} / \mathrm{s}]}\end{array}$ & $\mathrm{Re}_{\mathrm{j}}$ & \multicolumn{9}{|c|}{ Impact velocity $\mathrm{v}_{\mathrm{i}}[\mathrm{m} / \mathrm{s}]$} \\
\hline 0.88 & $\begin{array}{c}530 \\
5\end{array}$ & 0.99 & 1.13 & 1.33 & 1.50 & 1.66 & 1.80 & 1.93 & 2.05 & 2.17 \\
\hline 0.98 & $\begin{array}{c}589 \\
5\end{array}$ & 1.08 & 1.21 & 1.40 & 1.56 & 1.71 & 1.85 & 1.98 & 2.10 & 2.21 \\
\hline 1.08 & $\begin{array}{c}648 \\
4\end{array}$ & 1.17 & 1.29 & 1.47 & 1.62 & 1.77 & 1.90 & 2.03 & 2.15 & 2.26 \\
\hline 1.18 & $\begin{array}{c}707 \\
4\end{array}$ & 1.26 & 1.37 & 1.54 & 1.69 & 1.83 & 1.96 & 2.08 & 2.20 & 2.31 \\
\hline 1.28 & $\begin{array}{c}766 \\
3 \\
\end{array}$ & 1.35 & 1.46 & 1.62 & 1.76 & 1.90 & 2.02 & 2.14 & 2.25 & 2.36 \\
\hline 1.38 & $\begin{array}{c}825 \\
2\end{array}$ & 1.44 & 1.54 & 1.69 & 1.83 & 1.96 & 2.08 & 2.20 & 2.31 & 2.41 \\
\hline 1.47 & $\begin{array}{c}884 \\
2\end{array}$ & 1.54 & 1.63 & 1.78 & 1.91 & 2.03 & 2.15 & 2.26 & 2.37 & 2.47 \\
\hline 1.57 & $\begin{array}{c}943 \\
1\end{array}$ & 1.63 & 1.72 & 1.86 & 1.99 & 2.11 & 2.22 & 2.33 & 2.43 & 2.53 \\
\hline 1.67 & $\begin{array}{c}100 \\
21\end{array}$ & 1.73 & 1.81 & 1.94 & 2.06 & 2.18 & 2.29 & 2.39 & 2.49 & 2.59 \\
\hline 1.77 & $\begin{array}{c}106 \\
10\end{array}$ & 1.82 & 1.90 & 2.03 & 2.14 & 2.26 & 2.36 & 2.46 & 2.56 & 2.66 \\
\hline 1.87 & $\begin{array}{c}112 \\
00\end{array}$ & 1.92 & 1.99 & 2.11 & 2.23 & 2.33 & 2.44 & 2.54 & 2.63 & 2.72 \\
\hline 1.96 & $\begin{array}{c}117 \\
89\end{array}$ & 2.01 & 2.09 & 2.20 & 2.31 & 2.41 & 2.51 & 2.61 & 2.70 & 2.79 \\
\hline 2.06 & $\begin{array}{c}123 \\
79\end{array}$ & 2.11 & 2.18 & 2.29 & 2.39 & 2.49 & 2.59 & 2.68 & 2.77 & 2.86 \\
\hline 2.16 & $\begin{array}{c}129 \\
68\end{array}$ & 2.21 & 2.27 & 2.38 & 2.48 & 2.58 & 2.67 & 2.76 & 2.85 & 2.93 \\
\hline
\end{tabular}

- The first entrainment regime is the one where during the impingement no bubbles appear; although for all our experimental conditions we had a fully turbulent flow developed in the nozzle ( $\mathrm{Re}>2300)$.

- The second one is the medium entrainment regime, where either only one or two bubbles appear after a period of time, remain trapped under the surface for a short time and disappear after a few seconds (incipient entrainment), or a swarm of bubbles is being entrained from time to time (intermittent entrainment).

- The last entrainment regime is that of continuous air entrainment, when large air pockets and bubble swarms are continuously being entrained. 


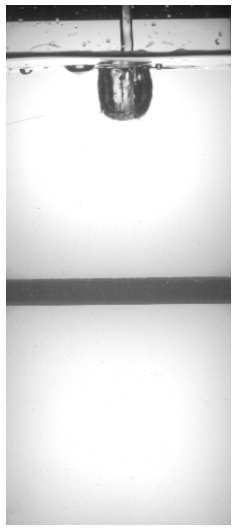

a.

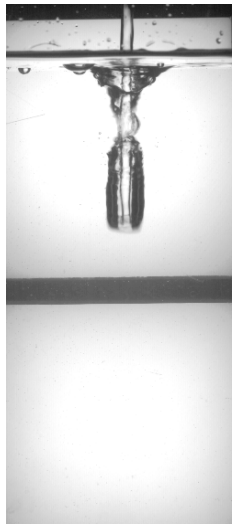

b.

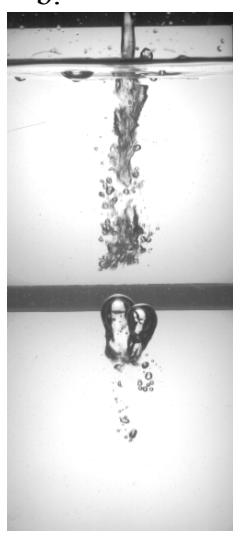

e.

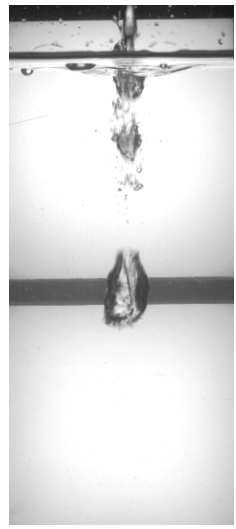

c.

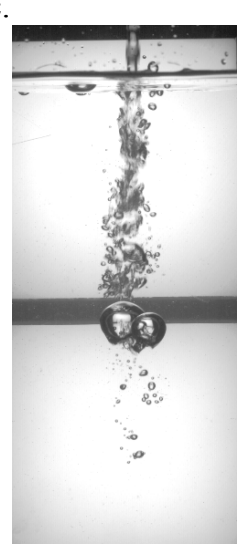

f.

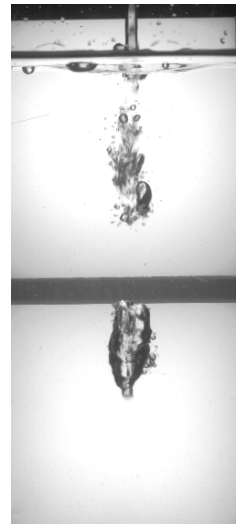

d.

Figure 2: Development of the air meniscus and its breakage at a jet impact velocity of $2.3 \mathrm{~m} / \mathrm{s}$. The times are, from a. to f., $15 \mathrm{~ms}, 55 \mathrm{~ms}, 85 \mathrm{~ms}$, $115 \mathrm{~ms}, 145 \mathrm{~ms}, 160 \mathrm{~ms}$; $0 \mathrm{~ms}$ being considered at the moment when the jet makes first contact with the water pool.

Figure 2 shows the occurrence of air entrainment. At the moment of the impact between the jet and the pool, an air meniscus forms around the jet at the plunging point and the pool surface curves downward in the form of an inverted meniscus. As the meniscus moves forward, toward the bottom of the tank, it stops growing (c) and the jet water begins to break through its' bottom (d). As the jet water moves downwards, the void retracts towards the free surface. According to [11], the water from the jet is found around the wall of the void and during the penetration it does not mix with the water from the pool; instead it forms a layer around the void and separates it from the surrounding water. This observation was possible due to the fact that dyed water was used for the jet.

Figure 3 illustrates the distribution of the entrainment regimes dependent on impact velocity and jet length for the experimental points of this work. 


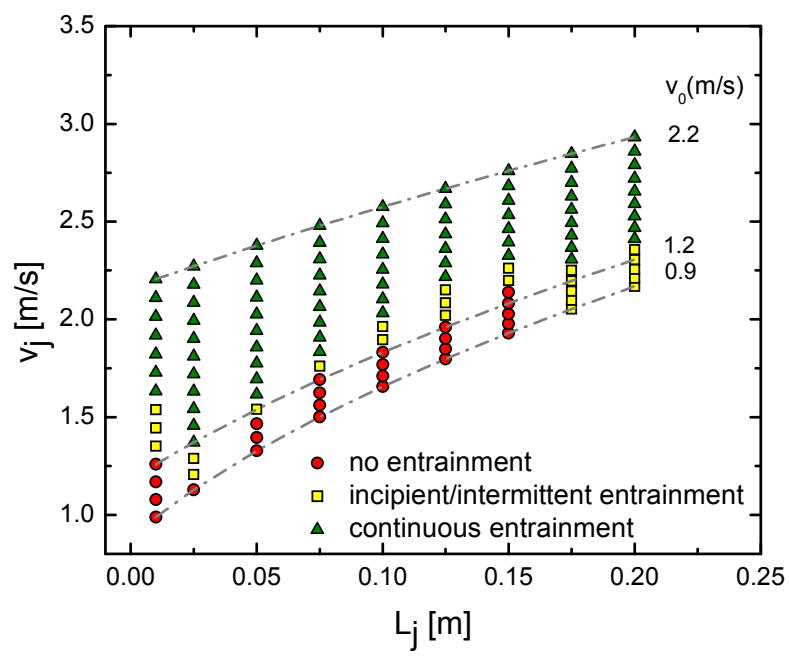

Figure 3: Entrainment regimes as a function of $\mathrm{v}_{\mathrm{j}}$, the jet impact velocity and the jet length, $\mathrm{L}_{\mathrm{j}}$.
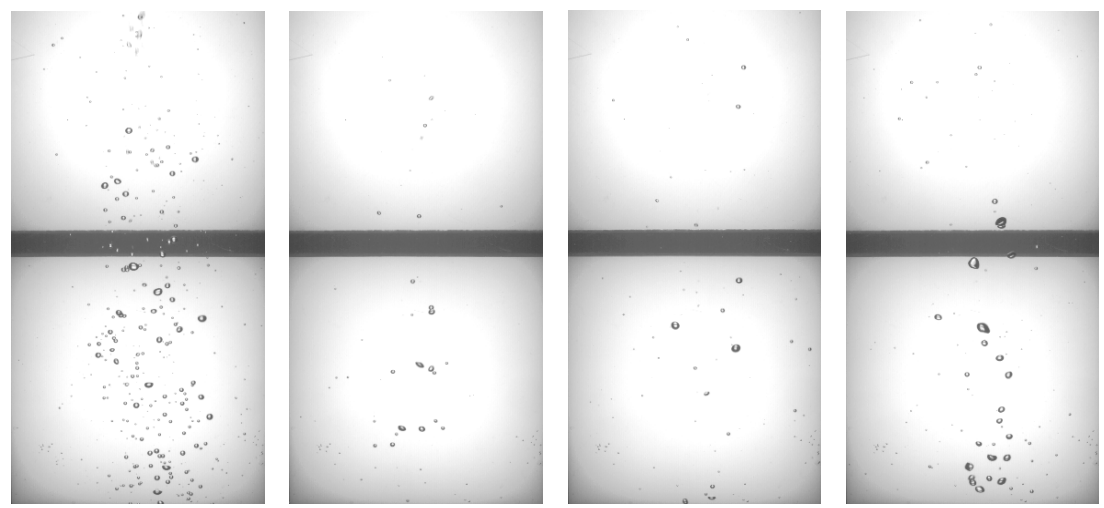

Figure 4: $\quad$ Air entrainment at $\mathrm{v}_{0}=1.18 \mathrm{~m} / \mathrm{s}$, for $\mathrm{L}_{\mathrm{j}}=0.025 \mathrm{~m} ; 0.05 \mathrm{~m} ; 0.175 \mathrm{~m}$; $0.2 \mathrm{~m}$ (from left to right).

In the case of turbulent vertical liquid jets, the surface tension forces no longer dominate the process of keeping the surface of the jet smooth. Due to the friction between the liquid and the gas, the surface of the jet becomes rough and bumpy.

The length of the jet along with the gravitational acceleration plays an important role in the inception of air entrainment. For different flow rates, air entrainment does not occur. We observed that as soon as the length of the jet is being increased even with just a few millimeters, air entrainment occurs. 
A distinctive and interesting situation takes place for $\mathrm{v}_{0}=1.2 \mathrm{~m} / \mathrm{s}$, where all four entrainment regimes are present. They vary with the jet length, starting with no entrainment, then jumping directly to the continuous entrainment regime, switching to incipient/intermittent entrainment followed by no entrainment for several jet lengths, returning to intermittent entrainment and continuous entrainment. Figure 4 presents the pictures corresponding to the jet lengths for which air entrainment takes place.

\section{Methodology and analysis}

The objective of our experiments was to gather information about the bubble size distribution, the penetration depth of the plume, the width of the plume and their variation in time.

In each experiment a sequence of 2000 frames was recorded for different flow rates and lengths of the jet. Image processing algorithms were applied to extract the sought information.

\subsection{Penetration depth}

During the experiments, we observed that the length of the jet plays an important role in the entrainment process. We determined the penetration depths' variation in time for different experimental conditions. Due to the fact that the plume fluctuates, one cannot refer to the penetration depth at a distinct moment. Therefore, to find the penetration depth for a set of experimental conditions, the best way is to average it over the entire sequence.

Images were subdued to background subtraction, removal of the small air bubbles at the walls and averaging. Our algorithm averages over the entire 2000 frames sequence. It is noticeable in the averaged imagine (Fig. 5, bottom) that, at the tip, the plume is rarified, meaning that only a small amount of bubbles travels that deep. Therefore, we have chosen to consider as penetration depth the first grey level value from the bottom of the plume which represents only ten percent of the maximum grey level value of the averaged plume, found usually in the core of the plume.

Figure 5 shows an example of the penetration depth variation for two different flow rates, but identical impact velocity, $\mathrm{v}_{\mathrm{j}}=2.5 \mathrm{~m} / \mathrm{s}$. The penetration depth of the air bubbles decreases with the increase of the impact velocity, most of the time. The plume becomes denser with the velocity and, due to the fact that the buoyancy forces surpass the viscous drag exerted by the downward moving jet, the plume retracts towards the surface of the water.

According to Bin [1], the penetration depth can be calculated as:

$$
H_{p}=2.1 v_{j}^{0.775} d_{0}^{0.67}
$$

which means that for constant impact velocity, the penetration depth should be constant. The penetration depth as a result of eq. 2 is found between $12 \mathrm{~cm}$ and $16 \mathrm{~cm}$ for impact velocities between $2 \mathrm{~m} / \mathrm{s}$ and $3 \mathrm{~m} / \mathrm{s}$. Figure 6 shows the variation of the penetration depth for three different nozzle exit velocities and different jet lengths, as a result of our image processing algorithms. We observe 
that the behavior of the penetration depth is not monotonous with the impact velocity, opposite to eq. 2. However, the values of the penetration depth are found in the range given by Bin's formula, although we get different penetration depths for the same impact velocity and nozzle diameter, but different nozzle velocities and jet lengths. In our experiments we vary the impact velocity by modifying the length of the jet and the nozzle exit velocity. We can observe that for $\mathrm{v}_{0}=2 \mathrm{~m} / \mathrm{s}$ and $\mathrm{v}_{0}=2.2 \mathrm{~m} / \mathrm{s}$, the penetration depth has a downward trend (see Figure 5). It first decreases with the jet length and then fluctuates slightly between 13 and $14 \mathrm{~cm}$. By increasing the jet length for a constant nozzle exit velocity, we increase the impact velocity and the momentum transport of the jet into the pool. This should lead to a higher penetration depth, as predicted by eq. 2. In our case, the penetration depth does not increase with the impact velocity.

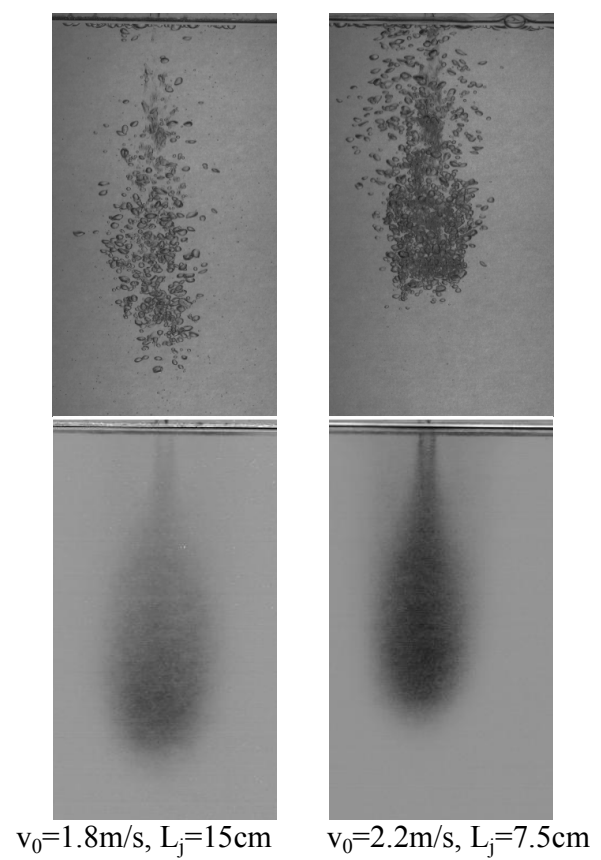

Figure 5: Example of the penetration depth variation for constant impact velocity $\mathrm{v}_{\mathrm{j}}=2.5 \mathrm{~m} / \mathrm{s}$, for the two different nozzle exit velocities and jet lengths: snapshots (top) and processed sequences (bottom).

This might be caused by higher gas void fractions in the plume which have been observed for higher jet lengths. High gas void fractions inside the plume counteract the jet momentum by buoyancy driven friction. Hence, the two opposite effects are responsible for the behavior of the penetration depth. However, in the case of $\mathrm{v}_{0}=1.8 \mathrm{~m} / \mathrm{s}$ the penetration depth behaves nonmonotonously, similar to the entrainment behavior in Figure 4. In Figure 4 $\left(\mathrm{v}_{0}=1.2 \mathrm{~m} / \mathrm{s}\right)$ the amount of entrained gas varies not monotonous with the jet length. This indicates that the jet surface instabilities which trigger gas 
entrainment do not increase monotonous with the jet length. Maybe different kinds of surface instabilities play a role here: surface instabilities triggered by the nozzle edge and surface instabilities which grow with the jet length, such as Rayleigh instabilities.

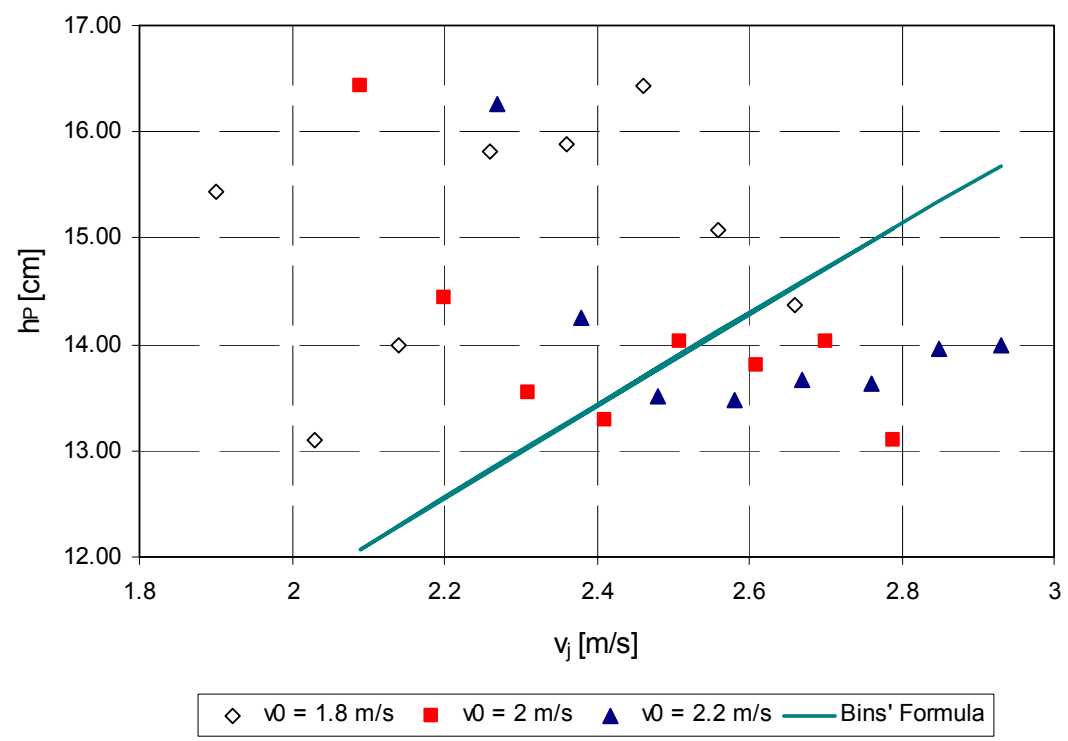

Figure 6: $\quad$ Processed penetration depth variation for three different nozzle exit velocities and jet length ranging between $0.025 \mathrm{~m}$ and $0.2 \mathrm{~m}$.

\subsection{Bubbles size distribution/estimation}

Two kinds of air bubbles are found in the plume: very small bubbles, which travel deeper into the tank, and bigger bubbles, which travel together towards the surface of the tank, in the form of a cone.

During their movement, the bubbles often change shape. Only the very small bubbles maintain their initial spherical form. The larger bubbles take an elliptical form which is also subdued to distortion. Even for higher jet velocities there are bubbles which escape from the cone and travel alone towards the surface. This happens frequently at the boundaries of the cone, where the buoyancy forces overcome the jet momentum. Coalescence and break-up phenomena were also observed.

Bubble sizes were estimated from single images by means of image processing through subsequent background subtraction, cell segmentation, bubble detection and bubble size calculation by means of a Hough transform based algorithm. It was found that the latter algorithm could only be used for experiments with a small number of bubbles in the plume. Otherwise, bubbles sizes could only be estimated at the edge of the plume where the bubbles do not overlap. Figure 7 shows an example of detected bubbles in a thin plume. 


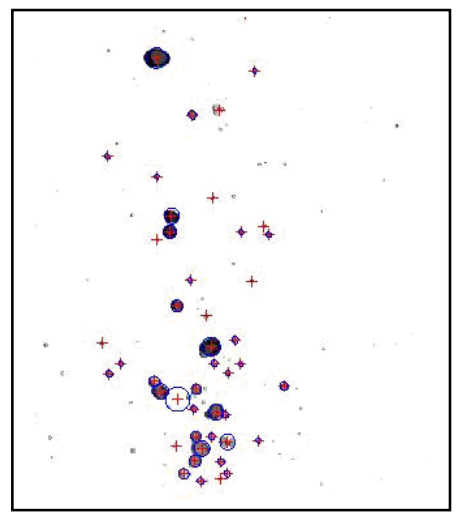

Figure 7: $\quad$ Image with bubbles detected (centre positions and radii marked).

The Sauter mean diameter in the region of the rising bubbles was about 3 $4 \mathrm{~mm}$, in agreement with previous data from the literature [1]. The smallest measurable bubbles were found to range between $0.3 \mathrm{~mm}$ and $1.5 \mathrm{~mm}$. Some of the largest bubbles had diameters around the value of $7 \mathrm{~mm}$.

\section{Conclusions}

To study the occurrence of air entrainment by means of impinging circular jets, we used a video observation technique. Experiments have been carried out in a square tank for various jet exit velocities and jet lengths. The experimental data was processed and will be used to validate the models implemented in CFD.

Some key parameters in air entrainment occurrence are: the velocity of the jet, the physical properties of the liquid, the length of the jet (falling height) and the turbulence of the jet. The penetration depth of the plume was measured and compared with an empirical formula by Bin. It has been found that in addition to the impact velocity, the amount of entrained gas has a significant effect on the penetration depth.

Further experiments are planned at FZD for a better observation of the liquid field velocities by PIV methods and quantitative void fraction measurements with $\mathrm{x}$-ray radiography.

\section{References}

[1] Bin A. K., Gas entrainment by plunging liquid jets. Chem. Eng. Sci., Vol. 48, No. 21, pp. 3585-3630, 1993.

[2] Bonetto F. \& Lahey Jr. R.T., An experimental study on air carryunder due to a plunging liquid jet. Int. J. Multiphase Flow, Vol. 19, No. 2, pp. 281294, 1993. 
[3] Chanson H., Aoki S. \& Hoque A., Physical modelling and similitude of air bubble entrainment at vertical circular plunging jets. Chem. Eng. Sci., Vol. 59, pp. 747-758, 2004.

[4] Chanson H. \& Brattberg T., Experimental investigations of air bubble entrainment in developing shear layers. Univ. of Queensland, Dep. of Civil Eng., Report CH48/97, 1997.

[5] Chanson H. \& Manasseh R., Air entrainment processes in a circular plunging jet: void-fraction and acoustic measurements. J. of Fluids Eng., Vol. 125, pp. 910-921, 2003.

[6] Chirichella D., Ledesma R. Gomez, Kiger K. T. \& Duncan J. H., Incipient air entrainment in a translating axisymmetric plunging laminar jet. Physics of Fluids, Vol. 14, pp. 781-790, 2002.

[7] Cummings P. D. \& Chanson H., An experimental study of individual air bubble entrainment at a planar plunging jet. Trans. I. Ch. E., Vol. 76, pp. 159-164, 1999.

[8] Davoust L., Achard J. L. \& El Hammoumi M., Air entrainment by a plunging jet: the dynamical roughness concept and its estimation by a light absorption technique. Int. Journal of Multiphase Flow, Vol. 28, pp. 1541$1564,2002$.

[9] El Hammoumi M., Achard J. L. \& Davoust L., Measurements of air entrainment by vertical plunging liquid jets. Experiments in Fluids, Vol. 32, pp. 624-638, 2002.

[10] Lin T. J. \& Donnelly H. G., Gas bubble entrainment by plunging laminar liquid jets. A. I. Ch. E. Journal, Vol.12, pp. 563-571, 1966.

[11] Oguz H. N., The role of surface disturbances in the entrainment of bubbles by a liquid jet. J. Fluid Mech., Vol.372, pp. 189-212, 1998.

[12] Soh W. K., Khoo B. C. \& Yuen W. Y. D., The entrainment of air by water jet impinging on a free surface. Exp. in Fluids, Vol. 39, pp. 496-504, 2005.

[13] Zhu Y., Oguz H. N. \& Prosperetti A., On the mechanism of air entrainment by liquid jets at a free surface. J. Fluid Mech., Vol.404, pp. 151-177, 2000. 